\title{
Viscoelastic Properties of Polymers. 4. Thermorheological Complexity of the Softening Dispersion in Polyisobutylene
}

\section{J. Plazek* and I.-C. Chay}

Department of Materials Science and Engineering, University of Pittsburgh, Pittsburgh, Pennsylvania 15261

\author{
K. L. Ngai and C. M. Roland
}

Naval Research Laboratory, Washington, D.C. 20375-5320

Received May 17, 1995; Revised Manuscript Received June 29, $1995^{\otimes}$

\begin{abstract}
The viscoelastic behavior of a high molecular weight polyisobutylene has been revisited to investigate the unique high-frequency peak (or shoulder) seen in the loss tangent behavior in the glass to rubber softening dispersion. Creep measurements made in the temperature range -74 to $+27{ }^{\circ} \mathrm{C}$ were initially reduced to a curve, which, upon transformation from the time to the frequency domains, yielded a loss tangent peak with no hint of any shoulder. The original creep compliance curves were rescrutinized independently, and slight variations in the derivatives of the curves revealed the elusive high-frequency loss peak. Additional dynamic measurements were made to connect the transformed creep data to some of the original Fitzgerald, Grandine, and Ferry data [J.Appl.Phys. 1953, 24, 911]. The combined results cover over 9 decades of frequency. This extensive range revealed that the mechanisms contributing to the high-frequency peak have a different temperature dependence than that of those contributing to the main loss peak.
\end{abstract}

\section{Historical Background}

The development of methods for studying the viscoelastic behavior of polymers began during the 1940 s and were pursued rather intensively into the $1950 \mathrm{~s}$. Polyisobutylene, a readily available saturated hydrocarbon with good stability, was the most studied polymer during this time. A problem in the early days was comparing data obtained by different laboratories. It was unclear how results might vary from sample to sample, not due to experimental uncertainties, but because of a dependence on molecular weight and its distribution and on chain architecture. To establish a benchmark for the comparison of results extending over a time and frequency range not available in any single laboratory, Robert Marvin of the U.S. National Bureau of Standards obtained a 100-lb sample of a high molecular weight $\left(M_{\mathrm{w}}=1.56 \times 10^{6}\right)$ regular production polyisobutylene (PIB) from the Esso Standard Oil Co. Tests were made to check the uniformity of the material, and in 1949 samples were sent to 27 laboratories in several countries. These labs where equipped to cover different ranges of time and frequency, yielding results which covered 15 decades of the time/frequency scale. ${ }^{1}$ Times are equivalent to the reciprocal circular frequency $\omega(\mathrm{rad} / \mathrm{s}),=2 \pi v$, where $v$ is the frequency in hertz (cycles/s).

The majority of the measurements were made in an oscillatory mode, yielding complex dynamic moduli or compliances in the frequency domain. There were also two sets of stress relaxation measurements and one creep study. The results from seven of the laboratories were equal within a $10 \%$ spread. These were combined by Marvin to show for the first time how the components of the dynamic moduli and compliances of a high molecular linear polymer varied from the glassy level of response at high frequencies to the approach to steady-state behavior at low frequency.

At the time it was not completely known how the features of the modulus and compliance curves pre-

\footnotetext{
${ }^{\otimes}$ Abstract published in Advance ACS Abstracts, August 15, 1995
}

sented in Marvin's summary paper varied with parameters such as molecular weight and its distribution. However, earlier work on PIB by Brown and Tobolsky ${ }^{2}$ established that the stress relaxation modulus in the glass to rubberlike dispersion was sensibly independent of molecular weight. For high molecular weight samples a rubberlike plateau was observed as a function of logarithmic time. In further studies on PIB Andrews and Tobolsky ${ }^{3}$ found the terminal relaxation time to be proportional to the 3.3 power of molecular weight. The same molecular weight dependence for the viscosity had previously been established by a number of studies, ${ }^{4}$ including those of Fox and Flory on PIB. Of course, the 3.3 or 3.4 exponential behavior has since been documented to hold for virtually all linear polymers.

Viscoelastic mechanisms before and after the rubberlike plateau give rise to two peaks in the relaxation and retardation spectra, with that at the longer times having the same temperature and molecular weight dependences as those of the viscosity, ${ }^{5,6}$ while the short-time group of mechanisms clearly has a more intense temperature dependence for most linear polymers. This difference in temperature dependences is minimal for PIB. ${ }^{7,8}$ For low molecular weight PIB's (e.g., 500050 000) Leaderman, Smith, and Jones ${ }^{9}$ found that the short-time/low-temperature recoverable creep compliance showed only one apparent dispersion from the glassy level (at ca. $10^{-9} \mathrm{~Pa}^{-1}$ ) up to a steady-state level $\left(\sim 10^{-5} \mathrm{~Pa}^{-1}\right)$. Only a single peak in the retardation spectrum was observed. The entanglement network was not sufficiently developed to give rise to a delayed group of mechanisms following a transient rubbery plateau. Leaderman, Smith, and Williams ${ }^{10}$ discovered that the molecular weight distribution has an enormous effect on the terminal viscoelastic effect. At the time PIB samples with narrow molecular weight distributions could not be made, so the dependence of the steady-state recoverable compliance $J_{\mathrm{e}}$ on molecular weight could not be established.

Dynamic mechanical measurements made on the widely distributed U.S. National Bureau of Standards 


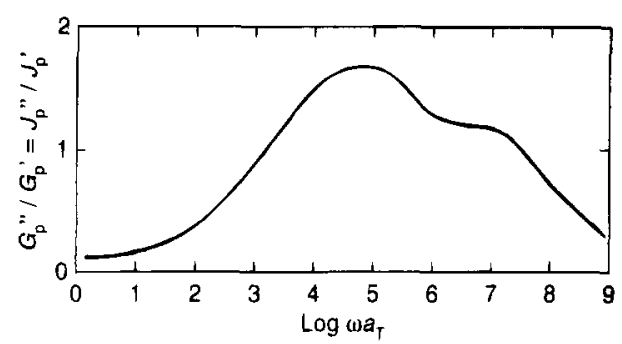

Figure 1. Reduced loss tangent, $\tan \delta\left(G^{\prime \prime} / G^{\prime}=J^{\prime \prime} / J^{\prime}\right)$, as a function of reduced frequency obtained by Ferry, Grandine, and Fitzgerald. ${ }^{16}$ Reference temperature $T_{0}=25^{\circ} \mathrm{C}$.

PIB sample by Fritzgerald, Grandine, and Ferry showed ${ }^{11,12}$ a feature that remains unique even today among linear polymers. Their reduced dynamic data revealed an asymmetrical loss tangent peak with a prominent shoulder at high reduced frequencies. This result, being of central importance to our present work, is reproduced in Figure 1. Interestingly, this shoulder is not indicated in the recoverable creep compliance curves obtained by Leaderman et al.$^{9,10}$ It should be noted that, along with the stress relaxation data of Andrews and Tobolsky, ${ }^{3}$ the dynamic compliance results of Fitzgerald, Grandine, and Ferry ${ }^{11,12}$ represent the earliest and best evidence for the time-temperature reduction process, which yields an extended frequency or time scale curve.

Recently, in the first two papers of this series, $7,8,13$ we investigated thoroughly the thermorheological properties of both high molecular weight and low molecular weight PIB and compared them with that of polystyrene (PS), another well-studied amorphous polymer. These measurements were motivated by the contrasting thermorheological properties of PIB and PS. A large difference of viscoelastic responses of PIB and PS is expected by the coupling theory ${ }^{7,8}$ based on the difference in their capacities for intermolecular coupling. The experimental data obtained in refs 7 and 8 were in agreement with these expectations and have since spurred on continued study of PIB.

\section{Introduction}

Polyisobutylene exhibits several properties which distinguish it from other flexible-chain polymers. This is ironic given the many contributions, as described above, PIB has made to our general understanding of polymer viscoelasticity. The most conspicuous of PIB's peculiarities include the following:

(i) The segmental relaxation dispersion of PIB is unusually narrow. When the relaxation function measured by dynamic light scattering ${ }^{14}$ is fitted to the Kohlrausch function ${ }^{15}$

$$
G(t)=G_{0} \exp \left[-(t / \tau)^{\beta}\right]
$$

The stretch exponent $\beta$ is found to be equal to 0.55 , whereas for most polymers $\beta<0.50$. The large value of $\beta$ for PIB is also deduced from quasi-elastic neutron scattering experiments. ${ }^{16}$

(ii) A comparison of the temperature dependence of the local segmental relaxation time $\tau$ of polymers was made ${ }^{17,18}$ by plotting $\log \tau$ against $T_{\mathrm{g}} / T$ where $T_{\mathrm{g}}$ is the temperature at which $\tau$ attains a value of $100 \mathrm{~s}$. Among amorphous polymers PIB exhibits the weakest normalized temperature $\left(T_{\mathrm{g}} / T\right)$ dependence. A strong inverse correlation between the $\left(T_{\mathrm{g}} / T\right)$ dependence and $\beta^{17-20}$ also indicates that PIB has a large $\beta$. (iii) Notwithstanding the large stretch exponent for the segmental relaxation function determined from light and quasi-elastic neutron scattering, the dispersion in PIB's dynamic mechanical loss modulus or recoverable creep compliance is quite broad. ${ }^{11,12,19,20}$ An analysis of the behavior of PIB in the glass transition zone led to the suggestion ${ }^{20}$ that other modes, transpiring over longer length scales than that of the segmental motion, were contributing to the observed mechanical response. In fact, in a low molecular weight PIB new modes situated in the time scale between the Rouse modes and the local segmental motion were directly observed by photon correlation spectroscopy in the retardation time spectrum obtained by the inverse Laplace transformation of the time correlation function. ${ }^{14}$

(iv) The glass-rubber transition zone of PIB is significantly broader than that of most other polymers. This is reflected by the small value of 0.65 of its "steepness index", defined as the maximum (absolute) value of $\mathrm{d} \log (G(t))$ vs $\mathrm{d} \log (t)$ in the glass-rubber transition region. ${ }^{21}$ For most polymers steepness indices are larger, for example, 1.57 for polystyrene.

(v) The terminal and the segmental relaxations of many high molecular weight polymers, including PS, when investigated in a common temperature range have different temperature dependences. ${ }^{22-25}$ However, this difference is not present in the behavior of PIB. ${ }^{8}$ Moreover, in many polymers of low molecular weight the steady-state compliance $J_{\mathrm{e}}$ has been found to decrease drastically as temperature decreases toward $T_{\mathrm{g} .}{ }^{13,26}$ The size of this effect is much reduced in PIB. ${ }^{8}$

(vi) Polymers generally exhibit a single maximum in their loss tangent versus either frequency or temperature,${ }^{27}$ however, as first discovered by Fitzgerald, Grandine, and Ferry ${ }^{11,12}$ (FGF), the dynamic mechanical loss tangent of PIB has an additional shoulder on the highfrequency (or low-temperature) side of the peak. This two-peak structure of tan $\delta$ is likely related to the two peaks found in the retardation spectrum obtained by photon correlation spectroscopy mentioned in iii.

Item vi is the focus of the present study. Additional mechanical measurements at longer times/lower frequencies have been made by us on PIB, in order to clarify the origin of the unique two-peak structure of $\tan \delta$ previously seen at higher frequencies by FGF. As referred to in $\mathrm{v}$, among amorphous polymers PIB shows the least degree of departure from thermorheological simplicity. In this paper we address the question of whether the (unusual) shape of the loss tangent versus frequency curve from PIB is sensitive to temperature.

\section{Experimental Section}

The PIB used in this study was from the original 1940s batch distributed by Marvin from the U.S. National Bureau of Standards, NBS (now NIST). This is the same PIB as studied by Fitzgerald, Grandine, and Ferry. The sample will, henceforth, be referred to as NBS-PIB. The absence of unsaturation makes the polymer quite resistant to degradation. Creep measurements of its terminal zone behavior verified that the material has not changed over the past 50 years. In any case, our interest in the present work is limited to the glass to rubber softening dispersion.

The creep experiments were carried out with a magnetic bearing torsional creep apparatus that has been described elsewhere. ${ }^{28}$ The dynamic mechanical measurements were obtained with a Bohlin VOR rheometer. A parallel plate geometry was used for both experiments.

\section{Results}

In recent years the creep compliance behavior of several PIB samples with differing molecular weights 


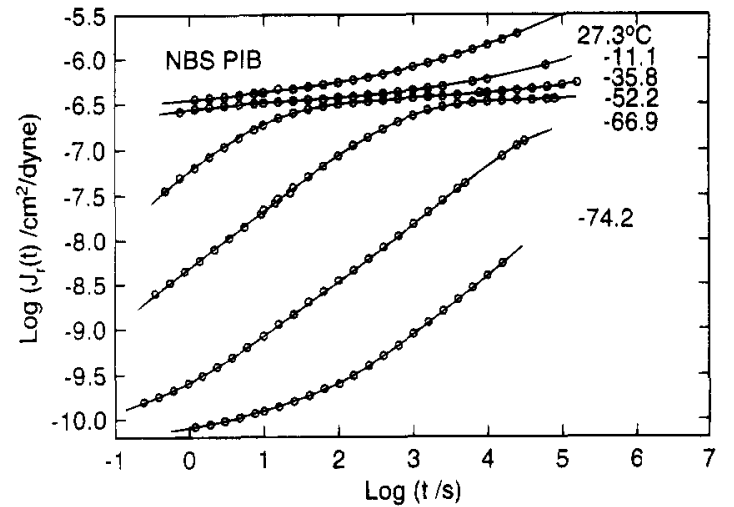

Figure 2. Double-logarithmic plot of the recoverable compliance $J_{\mathrm{r}}(\mathrm{t})\left(\mathrm{cm}^{2} /\right.$ dyn $)$ of the National Bureau of Standards polyisobutylene sample measured as a function of time at six temperatures as indicated.

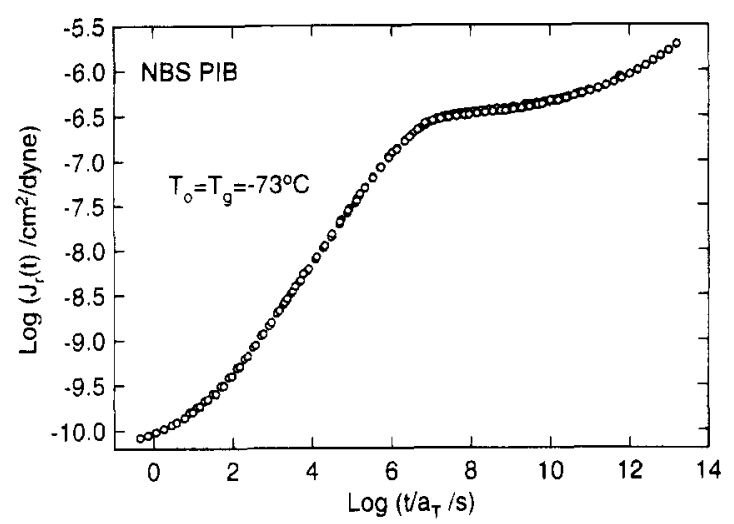

Figure 3. Data from Figure 2 reduced to a common curve at the reference temperature $T_{0}$ of $-73^{\circ} \mathrm{C}$.

has been determined. Compliances from the glassy level out to the terminal (steady-state) zone were measured. Only some of the results have been published. ${ }^{29}$ Surprisingly, when the results were transformed to the loss tangent, no high-frequency peak or shoulder was found. However, for at least a very high molecular weight PIB $\left(>10^{6}\right)$ its existence was certain. It had been observed not only by FGF but by one of us ${ }^{30}$ at the U.S. Naval Research Laboratory and much earlier by DeWitt, Zapas, and Markovitz at the Mellon Institute $^{31}$ All of these results were obtained by nonresonant forced-oscillation measurements. In an attempt to elucidate this dilemma, creep measurements were made on an available sample of the NBS-PIB. The recoverable compliance $J_{\mathrm{r}}(t)$ results obtained at six temperatures, from $+27^{\circ} \mathrm{C}$ down to $-74^{\circ} \mathrm{C}$, are shown in Figure 2. Simple temperature reduction of the log $J_{\mathrm{r}}(t)$ curves to a reference temperature $T_{0}=T_{\mathrm{g}}=-73$ ${ }^{\circ} \mathrm{C}$ was accomplished with only time-scale $\log a_{\mathrm{T}}$ shifts. Vertical shifts, in this temperature range, have been shown to be unnecessary. ${ }^{32}$ The resulting reduced curve is presented in Figure 3. It appears to be as successful as any such curves presented in the literature. This reduced recoverable compliance curve was analyzed by means of an iterative interaction scheme $e^{6}$ to obtain the retardation spectrum $L$ shown in Figure 4. Repeated estimates of $L$ are made until the input $J_{\mathrm{r}}(t)$ curve can be calculated by numerical integration.

The iteration is stopped when the calculated curve fits within the scatter of the input data. Beyond this point no further information can be extracted from the reduced curve. With the optimized $L$ other viscoelastic functions were calculated. A logarithmic presentation

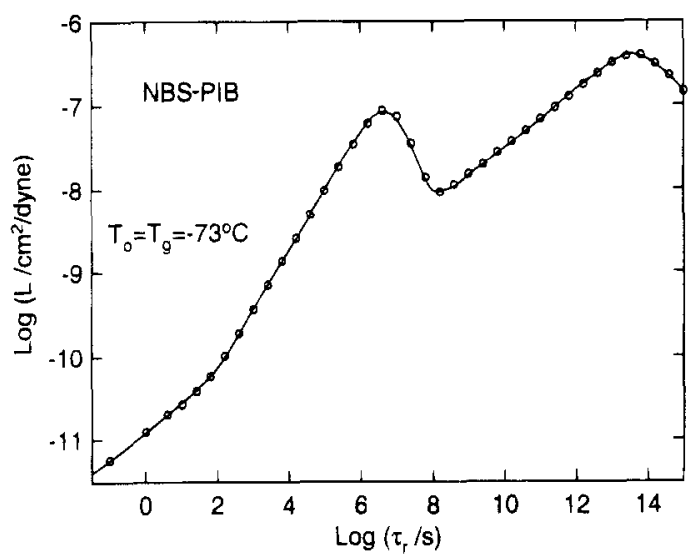

Figure 4. Logarithmic presentation of the retardation spectrum obtained from the reduced curve of Figure 2 as a function of the reduced retardation time $\tau_{\mathrm{r}}$.

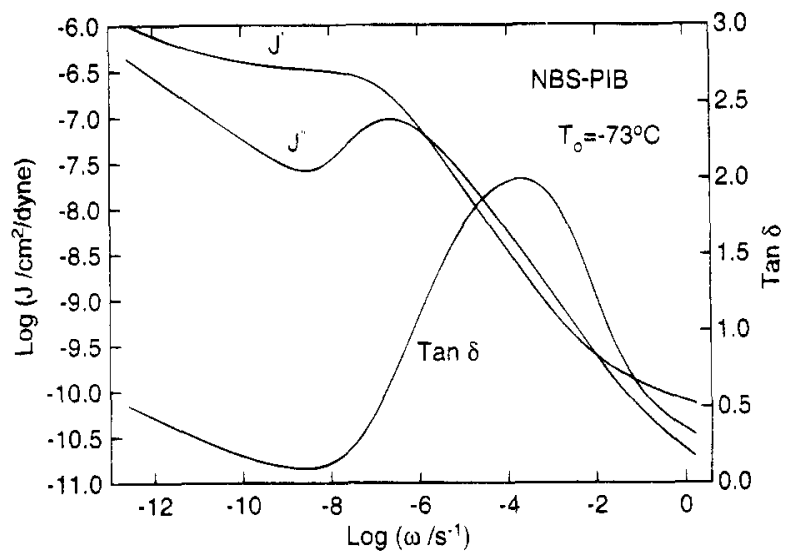

Figure 5. Logarithmic plot of the real, $J^{\prime}$, and imaginary, $J^{\prime \prime}$, components of the complex dynamic compliance as a function of the angular frequency $\omega=2 \pi \nu$, where $\nu$ is the frequency in hertz (cps). The linear loss tangent curve is shown as a function of $\log \omega$ at $-73^{\circ} \mathrm{C}$. These curves were calculated using the $L$ shown in Figure 4 .

of the storage $J^{\prime}$ and loss $J^{\prime \prime}$ dynamic compliances is given in Figure 5 along with a linear loss tangent curve as a function of the logarithmic reduced frequency $\omega$ $(\mathrm{rad} / \mathrm{s})$. The $\tan \delta$ peak, centered at $\log \omega=3.7$, shows no high-frequency shoulder. Chagrined at this result, we reexamined the original unreduced recoverable compliance curves. Derivatives of the $\log J_{\mathrm{r}}(t)$ vs $\log t$ $\equiv m$ were obtained manually and from a cubic spline function fit of the data points of the curves that are principally in the softening dispersion (i.e. $\log J_{\mathrm{r}}(t)<$ -6.5). The values obtained for $m$ are displayed in Figure 6. With these values and the recoverable creep compliances $J^{\prime}(\omega)$ and $J^{\prime \prime}(\omega)$ were calculated using the approximate relations ${ }^{33}$

$$
J^{\prime}(\omega) \cong[1-m(2 t)]^{0.8} J_{\mathrm{r}}(t)
$$

and

$$
J^{\prime \prime}(\omega)-\frac{1}{\omega \eta_{0}} \cong\left[m\left(\frac{2 t}{3}\right)\right]^{0.8} J_{\mathrm{r}}(t)
$$

where $\eta_{0}$ is the limiting low rate of shear viscosity coefficient; $m=\mathrm{d} \log J_{\mathrm{r}}(t) / \mathrm{d} \log t ; t=1 / \omega$ and $\tan \delta=$ $J^{\prime \prime} / J^{\prime}$. The $\tan \delta$ values calculated from the four $J_{\mathrm{r}}(t)$ curves obtained at $-34.8,-52.0,-66.9$, and $-74.2{ }^{\circ} \mathrm{C}$ are finally plotted in Figure 7 as data points represented by open and filled squares. The open circle data points on the $-35.8^{\circ} \mathrm{C}$ curve are those of FGF, obtained in 


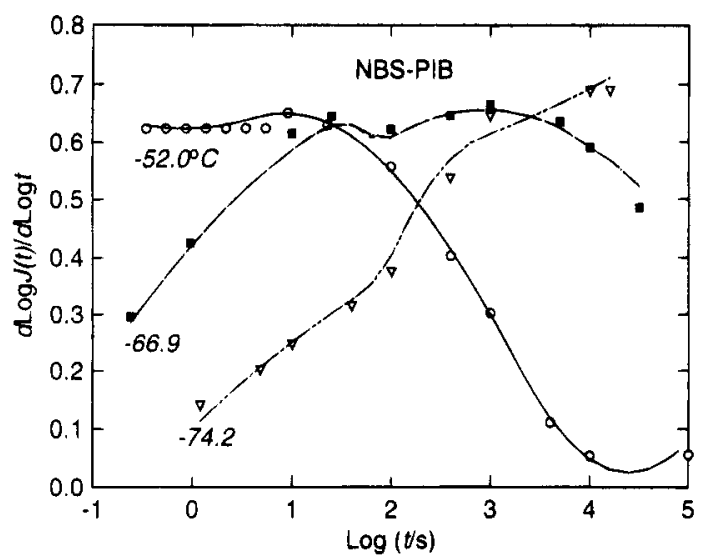

Figure 6. Derivatives obtained from the curves in Figure 2.

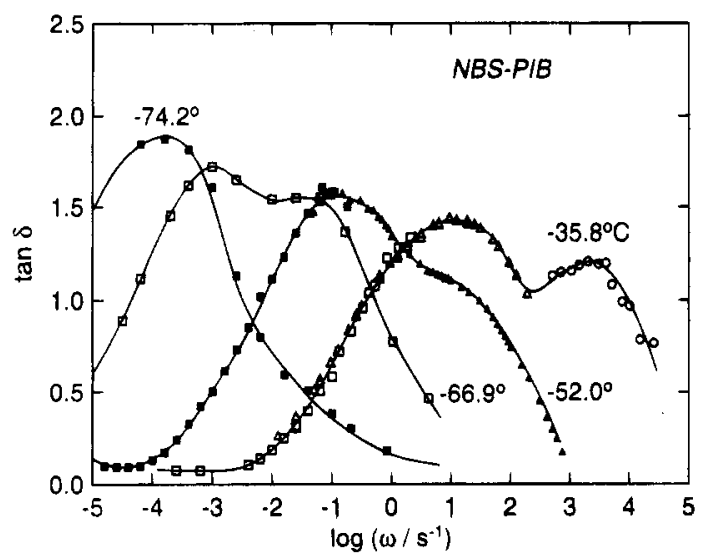

Figure 7. Loss tangent of NBS-PIB as a function of real frequency at the four indicated temperatures. The highest frequency data (open circles at $T=-35.8^{\circ} \mathrm{C}$ ) are from Fitzgerald, et al. ${ }^{15}$ The intermediate frequency results (open and filled triangles, respectively at $T=-52.0$ and $-35.8^{\circ} \mathrm{C}$ ) are from dynamic mechanical spectroscopy, while the lowfrequency data (filled, open, filled, and open squares respectively at $T=-74.2,-66.9,-52.0$, and $-35.8^{\circ} \mathrm{C}$ ) represent creep measurements.

nonresonant forced-oscillation measurements (Fitzgerald-Ferry instrument ${ }^{34}$ ). The intervening gaps between the creep data points and those of FGF were obtained from forced-oscillation measurements made with a Bohlin VOR dynamic mechanical rheometer (filled triangles at $-52.0^{\circ} \mathrm{C}$ and open triangles at -35 $\left.{ }^{\circ} \mathrm{C}\right)$.

The softening dispersion determined over a real frequency range of about 9 decades and over a wide temperature range (Figure 7) clearly confirms the existence of the two-peak structure. Moreover, the two mechanisms responsible for the main peak and the shoulder of $\tan \delta$ do not have the same temperature shift factors. The mechanisms contributing to the main peak have a weaker temperature dependence than the other mechanisms that give rise to the shoulder. The high-temperature peak or shoulder moves to lower frequencies faster than the main peak with decreasing temperatures. It is obvious that the high-frequency loss tangent peak was reduced out of existence, by the apparently successful superposition process.

\section{Conclusion}

From the data in Figure 7 we conclude that at least two groups of viscoelastic mechanisms, with different temperature dependences, contribute to the softening dispersion of the NBS-PIB. We can identify the primary maximum, occurring at the lower frequencies in Figure 1 , with the Rouse modes, which are known to dominate the viscoelastic response in the glass-rubber transition region. ${ }^{27}$ Rouse dynamics transpire over a length scale shorter than that associated with topological interactions (entanglements) but involve chain segments sufficiently long to be "Gaussian" (i.e., have a statistical end-to-end separation described by a Gaussian distribution function). It has been suggested that these chain segments contain on the order of 50 or more backbone bonds.

Identification of the higher frequency modes contributing to the loss tangent curves in Figure 1 is problematical. Segmental relaxation, associated with the glass to liquid transition, primarily involves correlated local motion of only a few backbone bonds. ${ }^{35,36}$ From published data on local segmental motion in $\mathrm{PIB}^{14}$ in another high molecular weight sample and considering that all but the lowest temperature curve in Figure 2 are at temperatures above the glass transition temperature $\left(=-73^{\circ} \mathrm{C}\right)$ of PIB, we expect local segmental relaxation to prevail at much higher frequencies than those associated with the satellite peak in Figure 7. Therefore, the mechanism giving rise to this highfrequency shoulder is neither Rouse nor segmental relaxation. Evidently, a third mode must be present.

Such intermediate dynamics, involving on the order of 10 backbone bonds, have previously been designated ${ }^{20}$ as "sub-Rouse modes" to convey that the motion involves segments, while larger than the couple of conformers involved in local segmental relaxation, containing fewer chain units than the shortest of the Gaussian submolecules described by the Rouse model. The actual number of backbone bonds involved in sub-Rouse modes is not known at this time. We assign the satellite peak in Figure 7 to these sub-Rouse dynamics. Of course, the length scales associated with the sub-Rouse modes are present in all polymers. The prominence of the subRouse modes in PIB is due to the unusual circumstance that the retardation times of its segmental motions have the weakest normalized temperature dependence among amorphous polymers (see ii in the Introduction). As a result, the encroachment of the PIB local segmental motions toward the Rouse modes occurs to a lesser degree than in other polymers. ${ }^{8}$ As a result, the separation in time scale between the segmental motion and the Rouse modes (which is a measure of the width of the softening dispersion) is considerably broader in PIB. The broad softening dispersion of PIB makes it easier for the sub-Rouse modes, situated in time within the dispersion, to be resolved as a shoulder in the loss tangent. In the usual circumstance (i.e., for other amorphous polymers), encroachment in time is more severe, which causes the width of the softening dispersion to become narrower. This narrower width makes the sub-Rouse modes more difficult to resolve in the loss tangent spectrum.

The anomalies of PIB listed in the Introduction, as well as the thermorheological complexity described herein, are experimental facts. Although clearly there must exist motions intermediate in length scale to the Rouse and local segmental modes, a detailed characterization, as well as identification, in polymers other than PIB, is lacking. Reflecting on the fact that recent investigations of PIB have contributed so greatly to understanding polymer viscoelasticity, one must conclude that after more than 50 years continued study of the viscoelastic behavior of amorphous polymers is still a worthwhile endeavor. 
It has been made clear that superposition of viscoelastic data to an apparently successful reduced curve is not sufficient proof of thermorheological simple behavior. Data extending over 8-10 decades of time and/or frequency appears to be necessary for a reasonable proof. The narrower frequency range of $2 \frac{1}{2}$ decades enabled Ferry, Grandine, and Fitzgerald ${ }^{12}$ to achieve apparently successful reduction of their data on the NBS-PIB.

Acknowledgment. The work at NRL was supported by the Office of Naval Research. K.L.N. is supported in part by ONR Contract N000149520203.

\section{References and Notes}

(1) Marvin, R. S. In Proceedings of the 2nd International Congress on Rheology; Harrison, V. G. W., Ed.; Oxford, U.K., July 1953; Butterworths: London, 1954.

(2) Brown, G. M.; Tobolsky, A. V. J. Polym. Sci. 1951, 6, 165.

(3) Andrews, R. D.; Tobolsky, A. V. J. Polym. Sci. 1951, 1, 221.

(4) Fox, T. G.; Loshaek, L. J. Appl. Phys. 1955, 26, 1080.

(5) Plazek, D. J. J. Phys. Chem. 1965, 69, 3480.

(6) Orbon, S. J.; Plazek, D. J. J. Polym. Sci., Polym. Phys. Ed. $1979,17,1871$.

(7) Plazek, D. J.; Zheng, X. D.; Ngai, K. L. Macromolecules 1992 , 25,4920

(8) Ngai, K. L.; Plazek, D. J.; Bero, C. A. Macromolecules 1993, 26,1065 .

(9) Leaderman, H.; Smith, R. G.; Jones, R. W. J. Polym. Sci. $1954,14,47$.

(10) Leaderman, H.; Smith, R. G.; Williams, L. C. J. Polym. Sci. 1959, 36, 233

(11) Fitzgerald, E. R.; Grandine, L. D., Jr.; Ferry, J. D. J. Appl. Phys. 1953, 24, 650 .

(12) Ferry, J. D.; Grandine, L. D., Jr.; Fitzgerald, E. R. J. Appl. Phys. 1953, 24, 911.

(13) The third paper in this series was published as: Plazek, D. J.; Bero, C. A.; Neumeister, S.; Floudas, G.; Fytas, G.; Ngai, K. L. Colloid Polym. Sci. 1994, 272, 1430.
(14) Rizos, A. K.; Jian, T.; Ngai, K. L. Macromolecules, in press.

(15) Kohlrausch, R. Pogg. Ann. Phys. (3) 1847, 12, 393; Pogg. Ann. Phys. (4) 1854, 1, 56, 179.

(16) Ngai, K. L.; Colmenero, J.; Alegria, A.; Arbe, A. Macromol. ecules 1992, 25, 6727 .

(17) Plazek, D. J.; Ngai, K. L. Macromolecules 1991, 24, 1222. McGrath, K.; Ngai, K. L.; Roland, C. M. Macromolecules 1995, 28,2825

(18) Böhmer, R.; Ngai, K. L.; Angell, C. A.; Plazek, D. J. J. Chem. Phys. 1993, 99, 4201.

(19) Catsiff, E.; Tobolsky, A. V. J. Colloid Sci. 1955, 10, 375.

(20) Santangelo, P. G.; Ngai, K. L.; Roland, C. M. Macromolecules 1993, 26, 2682.

(21) Aklonis, J. J.; Rele, V. B. J. Polym. Sci. C 1974, 46, 127.

(22) Plazek, D. J. J. Phys. Chem. 1965, 69, 3480.

(23) Plazek, D. J. Polym. J. 1980, 12, 43.

(24) Plazek, D. J.; Plazek, D. L. Macromolecules 1983, 16, 1469.

(25) Ngai, K. L.; Plazek, D. J. J. Polym. Sci., Polym. Phys. Ed. 1986, $24,619$.

(26) Plazek, D. J.; O'Rourke, M. V. J. Polym. Sci., Polym. Phys. Ed. 1971, 9, 209.

(27) Ferry, J. D. Viscoelastic Properties of Polymers; John Wiley \& Sons: New York, 1980.

(28) Plazek, D. J. In Methods of Experimental Physics; Fava, R. A., Ed.; Academic Press: New York, 1979; Vol. 16C, Chapter 11.

(29) Plazek, D. J.; Chay, I.-C., unpublished results.

(30) Roland, C. M., unpublished results.

(31) DeWitt, T. W.; Zapas, L. J.; Markovitz, H., unpublished results and personal communications.

(32) Plazek, D. J.; Chelko, A. J., Jr. Polymer 1977, 18, 15.

(33) Plazek, D. J.; Raghupathi, N.; Orbon, S. J. J. Rheol. 1979 , 23,477 .

(34) Fitzgerald, E. Phys. Rev. 1957, 108, 690. Fitzgerald, E.; Ferry, J. D. J. Colloid Sci. 1953, 8, 1.

(35) Adolf, D. B.; Ediger, M. D. Macromolecules 1992, 25, 1074.

(36) Bahar, I.; Erman, B.; Kremer, F.; Fischer, E. W. Macromolecules 1992, 25,816 .

MA950676S 